feeling hungry, tired, or stressed, and meeting family members' food preferences influenced their food shopping choices. The physical environment was also important, including product quality and variety, plus ease of accessing the store or products in-store. Some participants described taking a highly conscious approach to making food choices while shopping (i.e. lists), but many described how they and their family made unintended food selections as a result of prominent placement of unhealthy products. In addition to these six themes, participants described healthy eating as a personal responsibility but some stated that governments and supermarkets could be more supportive to help customers make healthier food shopping choices.

Conclusion This study identified a number of factors that influence women's food shopping choices and described how current placement strategies can shape these, particularly in a less aware manner. Creating healthier supermarket environments could produce a shared responsibility for healthy eating. Future research could further explore perceptions that healthy eating is a personal rather than a societal or business responsibility.

\section{OP08 UNDERSTANDING PRACTICES AND CHALLENGES OF (RE-)LOCALISING FOOD PRODUCTION AND CONSUMPTION IN SMALL ISLAND DEVELOPING STATES FOR BETTER NUTRITION: A QUALITATIVE MULTI-SITE STUDY}

${ }^{1} \mathrm{C}$ Guell ${ }^{*},{ }^{2} \mathrm{C}$ Brown, ${ }^{3} \mathrm{~V}$ lese, ${ }^{3} \mathrm{O}$ Navunicagi, ${ }^{3} \mathrm{M}$ Wairiu, ${ }^{1,4} \mathrm{~N}$ Unwin. ${ }^{1}$ European Centre for Environment and Human Health, University of Exeter Medical School, Truro, UK; ${ }^{2}$ George Alleyne Chronic Disease Research Centre, University of the West Indies, Bridgetown, Barbados; ${ }^{3}$ Pacific Centre for Environment and Sustainable Development, University of the South Pacific, Suva, Fiji; ${ }^{4}$ MRC Epidemiology Unit, University of Cambridge, Cambridge, UK

10.1136/jech-2020-SSMabstracts.8

Background Populations in Small Island Developing States have a high burden of malnutrition, including some of the highest rates globally of obesity and related non-communicable diseases. Underlying this burden is a growing reliance on low nutritional quality food imports, and improving food sovereignty and health through the production and consumption of local nutritious foods is therefore an urgent goal by national governments and regional organisations. As part of a larger project to develop evaluative methods, we aimed to explore factors affecting local food production and consumption, and communities' perceived impacts of local foods on health and socio-economic wellbeing in Fiji in the South Pacific and Saint Vincent and the Grenadines in the Caribbean.

Methods In the two settings, we conducted in total 52 indepth, semi-structured one-on-one interviews with key stakeholders involved in local food production, processing, trade and policy, as well as 28 focus groups with rural and urban, tenant and landowning communities of adult and young adult ages. Country teams and co-investigators jointly thematically analysed the transcripts using the software Dedoose. We placed emphasis on understanding commonalities and differences within and across contexts.

Results Across both settings in the Pacific and Caribbean, common concerns largely outweighed differences. Participants noted that while local food production was seen as essential to local nutrition policies for population health, government support was perceived to be limited, outdated (e.g. not recognising traditional agricultural methods) and funding declining rather than decreasing over the past decades. Local food producers saw themselves as marginalised against a backdrop of increasing preference for imported foods, yet also experienced an increasing interest and pride in local produce which potentially provides increased commercial opportunities. Narratives in the community focus groups contained corresponding tensions, highlighting the health and economic value of homeproduced foods, yet observing increasing social and generational changes towards buying and consuming processed foods. Many participants in both settings suggested that greater resilience could be achieved through cooperation and self-reliance on home food production but experienced many challenges related to environmental change such as extreme weather, pollution by pesticides and plastics, and to social conflicts and pressures such as limited access to land for home growing.

Conclusion Local community food production initiatives in Small Island Development States can be supported by understanding and addressing these complex challenges faced along the food value chain as well as by understanding local food practices, values and systems and their place in global networks.

On behalf of the CFaH Team

Funding Funded by the Global Challenges Research Fund through the UK Medical Research Council; Grant No. MR/ P025250/1.

\section{Wednesday 9 September}

\section{Session: Mental Health: Record Linkage}

\section{OP09 UNDERSTANDING THE MENTAL HEALTH OF CHILDREN KNOWN TO SOCIAL SERVICES IN NORTHERN IRELAND: AN ADMINISTRATIVE DATA LINKAGE STUDY}

S McKenna*, A Maguire, D O'Reilly. Centre for Public Health, Queen's University, Belfast, UK

\subsection{6/jech-2020-SSMabstracts.9}

Background Research suggests that children known to social services experience a higher prevalence of mental ill-health compared to their peers. However, previous studies have been limited based on sample sizes, comparison populations and level of detail around care interventions. Northern Ireland (NI) is unique as it has an integrated health and social care system holding data centrally on all individuals known to social services, allowing for a population wide examination of the mental health of children involved with social services and those never known. The aim of this project was to examine mental ill-health amongst a cohort of all children aged 18 years and under based on care exposure including Looked After Children (LAC) placed in foster/kinship/residential care, Children in Need (CIN) known to social services but not removed from home and those never known.

Methods This data linkage study linked population-wide primary care registration data to children's social care records, prescribed medication data and hospital records. Data were used to determine the period prevalence of mental ill-health in 2015 defined by receipt of psychotropic medication, psychiatric hospital admission and/or presentation to Emergency Department for self-harm. Logistic regression quantified the likelihood of each mental health outcome with adjustment for factors associated with mental ill-health and self-harm. 
Results The cohort consisted of 535,801 children aged 18 years or less in NI during 2015. Over 1 in $6(17.6 \%$, $\mathrm{n}=94,232$ ) were previously or currently known to social services. There was a clear stepwise association between level of interaction with social services and mental ill-health. Compared to children never known to social services LAC were nearly 9 times more likely to be in receipt of antidepressants $(\mathrm{OR}=8.93,95 \% \mathrm{CI} 7.06,11.29), 10$ times more likely to be in receipt of anxiolytics $(\mathrm{OR}=10.0695 \% \mathrm{CI}$ $7.28,13.92)$ and over 40 times more likely to be receipt of antipsychotics $(\mathrm{OR}=42.59$, 95\%CI 32.85, 55.23). Likelihood of presenting with self-harm and psychiatric hospital admission were also significantly higher in LAC $(\mathrm{OR}=24.42,95 \% \mathrm{CI} 17.16,34.73$ and $\mathrm{OR}=86.26,95 \% \mathrm{CI}$ 42.58, 174.75 respectively).

Conclusion Children known to social services have poorer mental health compared to those not known. The relationship is graded, with prevalence lowest for those never known, higher for children known as CIN, and highest for LAC. Additional analysis is underway exploring variations in mental health based on care pathways and reason known to social services.

\section{\begin{tabular}{|l|l}
\hline OP10 & SELF-REPORTED MENTAL HEALTH VERSUS
\end{tabular} PSYCHOTROPIC MEDICATION RECORD AS A PREDICTOR OF SUICIDE: A RECORD LINKAGE STUDY}

${ }^{1}$ IN Onyeka* , ${ }^{1} \mathrm{~A}$ Maguire, ${ }^{1,2} \mathrm{D}$ O'Reilly. ${ }^{1}$ Centre for Public Health, Queen's University Belfast, Belfast, UK; ${ }^{2}$ Admin Data Research Centre Northern Ireland, Centre for Public Health, Queen's University Belfast, Belfast, UK

\subsection{6/jech-2020-SSMabstracts. 10}

Background Both the rates of self-reported mental ill-health and the number of individuals in receipt of psychotropic medications are increasing across the UK. Research suggests that poor mental health is a major predictor of death by suicide, but measuring individual level mental ill-health can be difficult. Population-wide prescription medication records are available in many countries and could provide useful information on indicators of suicide risk, however, the association between self-reported mental health, medication record and suicide has not been well explored. This study aimed to assess if self-reported mental health, psychotropic medication record, or both in combination better predict risk of death by suicide.

Methods Data from the 2011 Northern Ireland Census on all individuals living in the community aged 18-74 years ( $n=1,098,967)$ were linked to the Enhanced Prescribing Database (EPD) and death registrations and followed up until end of 2015. Mental health status was ascertained through a single-item self-reported mental health question from the Census and/or a record of psychotropic medication in the EPD. Deaths by suicide were identified using ICD-10 codes. Logistic regression models were used to examine the association between measures of mental health and death by suicide, with adjustment for census-based individual attributes known to be associated with mental ill-health and suicide.

Results A total of 857 out of the 1,098,967 individuals died by suicide during the study period. The proportion who died by suicide was $0.05 \%$ in those without mental illhealth, $0.21 \%$ in those who self-reported poor mental health, $0.16 \%$ in those in receipt of psychotropic medication, and $0.38 \%$ in those with both self-report and psychotropic medication record. In the unadjusted logistic regression model, having both self-report and psychotropic medication record was the strongest predictor of suicide $(\mathrm{OR}=8.23$, 95\%CI: 6.97-9.72). Upon adjustment for sociodemographic characteristics and physical health morbidities, having both self-report and psychotropic medication record remained the strongest suicide predictor $(\mathrm{OR}=6.13,95 \% \mathrm{CI}$ : 4.94-7.61), followed by psychotropic medication record only $(\mathrm{OR}=4.00,95 \% \mathrm{CI}: 3.28-4.88)$ and self-report only $(\mathrm{OR}=2.88,95 \% \mathrm{CI}: 2.16-3.84)$.

Conclusion Individuals with both self-reported poor mental health and receipt of psychotropic medication had the highest likelihood of death by suicide. Of the two measures individually, receipt of psychotropic medication was associated with a higher likelihood of suicide mortality compared to selfreported mental health. Understanding which measures are the best indicators of suicide risk is vitally important for the effective targeting of interventions.

\section{OP11 ETHNICITY AS A KEY DRIVER OF SYSTEM-WIDE HEALTH AND CARE SERVICE COSTS IN PATIENTS WITH SERIOUS MENTAL ILLNESS: A LINKED ELECTRONIC HEALTH RECORD COHORT STUDY}

'S Winchester*, 2J Shand, "2 Sheringham, "J George. 'Institute of Health Informatics, University College London, London, UK; ${ }^{2}$ Institute of Epidemiology and Health Care, University College London, London, UK

\subsection{6/jech-2020-SSMabstracts. 11}

Background Improving care for people with serious mental illness (SMI) is a policy priority in England, yet little is known about service use patterns across the broader health and care system outside of acute and mental health care. We sought to investigate individual- and practice-level features associated with health and care system costs (as a comparable marker of use) for patients with SMI using a system-wide linked patient-level dataset that has been developed in Barking and Dagenham (B\&D), with the aim of driving improved inter-organisational working and financial sustainability.

Data This retrospective cohort study includes patients registered and resident in B\&D in financial years 2016/17 and 2017/18. GP electronic health records were linked with administrative data from community, acute and mental health Trusts serving the area for both years, and local authorityfunded social care records for 2016/17 (with costs imputed for 2017/18). The study population was adults on the SMI Quality and Outcomes Framework register, with comparator groups (1) matched on age, gender, and number of long term conditions (LTCs), and (2) the general adult population. Cost analyses took three forms: descriptive, regression at individuallevel (generalised linear model using a Tweedie distribution), and funnel plots at GP practice-level.

Results SMI patients had three-fold greater system costs than matched patients. Increased spending was concentrated in mental health Trust outpatient and social care settings. Increased spending was not observed in primary care and emergency departments. Apart from LTC count, ethnicity was the main driver of spending, particularly within social care. Spending per White British patient per year $(£ 10,181)$ was almost twice that of Asian or Black patients $(£ 5,442$ 